\title{
PARTIAL COMPLEMENTS IN FINITE GROUPS
}

\author{
INGRID CHEN \\ (Received 23 November 2008; accepted 6 January 2010) \\ Communicated by E. A. O’Brien
}

\begin{abstract}
Let $G$ be a finite group with normal subgroup $N$. A subgroup $K$ of $G$ is a partial complement of $N$ in $G$ if $N$ and $K$ intersect trivially. We study the partial complements of $N$ in the following case: $G$ is soluble, $N$ is a product of minimal normal subgroups of $G, N$ has a complement in $G$, and all such complements are $G$-conjugate.
\end{abstract}

2000 Mathematics subject classification: primary 20D10.

Keywords and phrases: soluble groups, complements, partial complements.

\section{Introduction}

Let $G$ be a finite soluble group with a normal subgroup $N$. A subgroup $H$ of $G$ is a complement of $N$ in $G$ if $H$ intersects $N$ trivially and $G=N H$. We define $K$ to be a partial complement of $N$ in $G$ if $K$ is a subgroup of $G$ and $K$ and $N$ intersect trivially. Consider the following question: if $G$ is a finite soluble group, when is each partial complement of $N$ in $G$ contained in a complement of $N$ in $G$ ? Hall [4] proved that if $G=N H$, where $N$ is a $p$-group ( $p$ a prime) and $p$ does not divide the order of $H$, then each partial complement of $N$ in $G$ is contained in a complement of $N$ in $G$.

Rose considered related problems in [6]. Assume that $p$ is a prime, $N$ is an abelian normal $p$-subgroup of $G$ and $P$ is a Sylow $p$-subgroup of $G$. He proves in [6, Theorem 4] that $P$ splits over $N$ and all the complements of $N$ in $P$ form a single conjugacy class in $P$ if and only if the following conditions hold: $G$ splits over $N$; all the complements of $N$ in $G$ form a single conjugacy class in $G$; and each partial complement of $N$ in $G$ is contained in some complement of $N$ in $G$. If $G$ is a finite primitive soluble group, then it splits over $N$ and all the complements of $N$ form a single conjugacy class in $G$. Rose gives examples of such groups $G$ where:

(i) each partial complement of $N$ is contained in a complement of $N$ [6, Theorem 11];

(C) 2010 Australian Mathematical Publishing Association Inc. 1446-7887/2010 \$16.00 
(ii) a partial complement of $N$ is not contained in a complement of $N$ [6, Example 16].

The problem was again raised by Doerk and Hawkes in [2] (see the discussion between Propositions 15.9 and 15.10). Like Rose, they provide an example [2, Example VIII, 2, 19] of a finite soluble primitive group $G$ where there is a partial complement of $N$ in $G$ that is not contained in any complement of $N$ in $G$.

Assume that $G$ is a finite soluble group, $N$ is a product of minimal normal subgroups of $G, N$ has a complement in $G$ and all its complements are conjugate. In this paper we give a necessary and sufficient condition for each partial complement of $N$ in $G$ to be contained in a complement of $N$ in $G$. Let $\operatorname{GF}(p)$ denote the field with $p$ elements.

THEOREM 1. Let $G$ be a finite soluble group. Let $N$ be a product of minimal normal subgroups of $G$ where $N$ is complemented in $G$ and all its complements are conjugate in $G$. Let $p$ be a prime and let $N_{p}$ be the Sylow p-subgroup of $N$. Then each partial complement of $N$ in $G$ is contained in some complement of $N$ in $G$ if and only if, for each prime $p$ dividing the order of $N, N_{p}$ is projective as a $\operatorname{GF}(p)(G / N)$-module.

\section{Preliminaries}

In this section we collect a number of results required in the proof of Theorem 1 . Notation is mostly standard; in general, we use the same notation as [2]. We assume throughout the paper that $p$ is a prime number.

LEMMA 2 [6, Lemma 9(i)]. Suppose $G$ is a finite group. Let $N$ be a normal subgroup of $G$ and let $H$ be a complement of $N$ in $G$. Then the number of conjugates of $H$ in $G$ is $|N| /\left|C_{N}(H)\right|$.

Let $F$ be a field of characteristic $p$ and let $K$ be a group of order $p$. We define an $F K$-module $U$ to be uniserial if the successive quotients of the radical series of $U$ are simple.

The following lemma is a consequence of [5, Theorem VII, 5.3] and its proof.

LEMMA 3. Let $F$ be a field of characteristic $p$ and let $K$ be a group of order $p$. Then the following hold.

(i) The regular $F K$-module is uniserial.

(ii) An FK-module generated by a single element is indecomposable.

(iii) $\operatorname{Rad}^{p-1}(F K) \neq 0$ and $\operatorname{Rad}^{p}(F K)=0$.

(iv) $\operatorname{Rad}^{i}(F K) / \operatorname{Rad}^{i+1}(F K)$ is simple, so $\operatorname{Rad}^{i}(F K) / \operatorname{Rad}^{i+1}(F K)$ has order $|F|$.

LEMMA 4. Let $F$ be a field of characteristic $p$ where $p$ is a prime. Let $K$ be a group of order $p$. Let the FK-module $V$ be the direct sum of $W_{1}$ and $W_{2}$ where $W_{1}$ contains no free submodule and $W_{2}$ is a free submodule or $W_{2}=0$. Then $\operatorname{Rad}^{p-1}(V)=0$ if and only if $W_{2}=0$. 
Proof. If $W_{2}=0$ then $V=W_{1}$. Let $U$ be an indecomposable submodule of $W_{1}$. By [5, Theorem VII, 5.3], there exists $0<i<p$ such that $U \cong F K / \operatorname{Rad}^{i}(F K)$. Therefore $\operatorname{Rad}^{p-1}(U)=0$. Since the radical of the direct sum of submodules is the sum of the radicals of each submodule, $\operatorname{Rad}^{p-1}(V)=0$.

If $W_{2} \neq 0$ then $\operatorname{Rad}^{p-1}\left(W_{2}\right) \neq 0$ since $W_{2}$ is free and $\operatorname{Rad}^{p-1}(F K) \neq 0$. Therefore $\operatorname{Rad}^{p-1}(V) \neq 0$. Note that in both cases, $\operatorname{Rad}^{p}(V)=0$.

In the following, we will deal with the semidirect product $G=V K$ where $K$ is a group of order $p$ and $V$ is an elementary abelian $p$-subgroup of $G$. Of course $V$ is a $\operatorname{GF}(p) K$-module, and we will denote by $\operatorname{Rad}(V)$ the radical of $V$ as a $\operatorname{GF}(p) K$ module so that we can apply the previous results; observe that $V$ is now viewed as a multiplicative group.

The next result is similar to [6, Lemma 9(ii)].

LEMMA 5. Let $G$ be the semidirect product of a normal elementary abelian subgroup $V$ and a cyclic subgroup $K$ of order $p$. Assume that $V=W_{1} \times W_{2}$ is the direct product of two $\mathrm{GF}(p) K$-submodules of $V$ such that $W_{1}$ contains no free submodule and $W_{2}$ is a free submodule. Then the elements of order $p$ in $G$ but not in $V$ are those of the form $k w$, where $w \in W_{1} \times \operatorname{Rad}\left(W_{2}\right)$ and $1 \neq k \in K$.

Proof. Let $k \in K$ and $w \in V$. Define $r_{1}=[w, k]$ and $r_{i+1}=\left[r_{i}, k\right]$. By induction,

$$
(k w)^{p}=k^{p} w^{p} r_{1}^{s_{1}} \cdots r_{p-2}^{s_{p-2}} r_{p-1} \quad \text { where } s_{i}=\left(\begin{array}{c}
p \\
i+1
\end{array}\right)
$$

for every $1 \leq j \leq p-2$. Note that if $w \in W_{1} \times \operatorname{Rad}^{j}\left(W_{2}\right)$ for any $0 \leq j \leq p$ then $r_{i} \in \operatorname{Rad}\left(W_{1}\right) \times \operatorname{Rad}^{j+i}\left(W_{2}\right)$. Moreover, $k^{p}=v^{p}=1$. On the other hand, since $r_{i} \in V$ and $p$ divides all the exponents $s_{i}$, then $r_{1}^{s_{1}} \cdots r_{p-2}^{s_{p-2}}=1$. Observe that $r_{p-1}=1$ if and only if $w \in W_{1} \times \operatorname{Rad}\left(W_{2}\right)$ (by Lemma 4). The result follows.

COROLlary 6. Let $K$ be a group of order $p$ where $p$ is a prime. Let the $\mathrm{GF}(p) K$ module $V$ be the direct product of $W_{1}$ and $W_{2}$. Assume that $W_{1}$ has order $p^{s}$ and does not contain any free module and that $W_{2}$ is a free module of rank $l$. Then the number of subgroups of order $p$ in VK that intersect $V$ trivially is $p^{s+l(p-1)}$.

PROOF. First we apply Lemma 5 to find the number of elements of order $p$ that are in $V K$ but not in $V$. Since any indecomposable module of $V$ is uniserial,

$$
\left|W_{1} \times \operatorname{Rad}\left(W_{2}\right)\right|=p^{s} p^{l(p-1)}=p^{l(p-1)+s} .
$$

So there are $\left(p^{l(p-1)+s}\right)(p-1)$ elements of order $p$ in $V K$ but not in $V$. Then the number of subgroups of order $p$ in $V K$ that intersect $V$ trivially is

$$
\frac{\left(p^{l(p-1)+s}\right)(p-1)}{p-1}=p^{l(p-1)+s} .
$$

This concludes the proof. 
COROLLARY 7. Let $G$ be the semidirect product $N K$, where $K$ is a subgroup of order $p$ and $N$ is an elementary abelian p-subgroup of $G$. Then the number of subgroups of order $p$ in $G$ that intersect $N$ trivially is equal to the number of conjugates of $K$ in $G$ if and only if $N$ is free as a $\mathrm{GF}(p) K$-module.

Proof. Let the $\operatorname{GF}(p) K$-module $N$ be the direct product of $W_{1}$ and $W_{2}$, where $W_{1}$ has order $p^{s}$ and does not contain any free module, and $W_{2}$ is a free module of rank $l$. By Corollary 6, the number of subgroups of order $p$ in $N K$ that intersect $N$ trivially is $p^{s+l(p-1)}$. Let $W_{1}=U_{1} \times \cdots \times U_{r}$, where $U_{i}$ are indecomposable submodules. Observe that $S_{i}$, the minimal submodule of $U_{i}$, is in the centralizer $C_{U_{i}}(K)$ and if $w \in U_{i}$ is not contained in $S_{i}$, then $w$ is not centralized by $K$. Therefore $\left|C_{U_{i}}(K)\right|=\left|S_{i}\right|=p$. Hence $\left|C_{W_{1}}(K)\right|=p^{r}$.

Similarly, $\left|C_{W_{2}}(K)\right|=p^{l}$. Hence $\left|C_{N}(K)\right|=p^{r} p^{l}=p^{l+r}$.

So, by Lemma 2, we obtain that the number of conjugates of $K$ in $N K$ is

$$
\frac{|N|}{\left|C_{N}(K)\right|}=\frac{p^{s+p l}}{p^{r+l}}=p^{s+l(p-1)-r} .
$$

Comparing this result with that of Corollary 6, we see that the number of subgroups of order $p$ in $N K$ that intersect $N$ trivially is the same as the number of conjugates of $K$ in $N K$ if and only if $r=0$; that is, if and only if $N$ is free as a $\operatorname{GF}(p) K$ module.

LEMMA 8. Let $G$ be a finite soluble group. Let $N$ be a product of minimal normal $p$-subgroups of $G$ where $p$ is a prime. Assume that $H$ is a complement of $N$ in $G$ and that all the complements of $N$ in $G$ are conjugates of $H$. Let $H_{0}$ be a subgroup of $H$ of order $p$. Then each subgroup of order $p$ in $\mathrm{NH}_{0}$ (the semidirect product) but not in $\mathrm{N}$ is contained in a complement of $N$ in $G$ if and only if the number of conjugates of $H_{0}$ in $\mathrm{NH}_{0}$ is equal to the number of subgroups of order $p$ that are in $\mathrm{NH}_{0}$ but not in $\mathrm{N}$.

PROOF. Let $L$ be a subgroup of order $p$ in $N H_{0}$ but not in $N$. Then $L$ is contained in a complement of $N$ in $G$ if and only if there exists an element $n$ in $N$ such that $L \subseteq N H_{0} \cap H^{n}$. Observe that $N H_{0}=\left(N H_{0}\right)^{n}$ for each $n \in N$. Hence $L \subseteq N H_{0} \cap H^{n}$ if and only if

$$
L \subseteq\left(N H_{0}\right)^{n} \cap H^{n}=\left(N H_{0} \cap H\right)^{n}
$$

However,

$$
\left(N H_{0} \cap H\right)^{n} \cong N^{n}\left(N H_{0} \cap H\right)^{n} / N^{n} \cong\left(N H_{0} \cap N H\right)^{n} / N^{n}=\left(N H_{0}\right)^{n} / N^{n} \cong H_{0}^{n} .
$$

Hence $\left|\left(N H_{0} \cap H\right)^{n}\right|=\left|H_{0}^{n}\right|=p$. Therefore $L \subseteq\left(N H_{0} \cap H\right)^{n}$ if and only if $L=H_{0}^{n}$. Hence each subgroup of order $p$ in $\mathrm{NH}_{0}$ but not in $N$ is contained in some complement of $N$ in $G$ if and only if the number of conjugates of $H_{0}$ in $N_{0}$ is equal to the number of subgroups of order $p$ that are in $\mathrm{NH}_{0}$ but not in $\mathrm{N}$. 
Before we prove Lemma 9, we first note a result needed in the proof. Let $H$ be a group, let $F$ be a finite field of prime characteristic $p$ and let $E$ be its algebraic closure. Let $V_{E}$ denote the $E H$-module $E \otimes_{F} V$ [5, Definition VII, 1.1]. By [5, Exercise VII. 7. 19], the $F H$-module $V$ is projective if and only if the $E H$-module $V_{E}$ is projective.

LEMMA 9. Let $F$ be a field of prime characteristic $p$. Let $H$ be a soluble group and let $V$ be a semisimple $F H$-module. Then $V$ restricted to each subgroup $C$ of $H$ of order $p$ is projective as a $F C$-module if and only if $V$ is projective.

PROOF. If $V$ is projective as a $\mathrm{GF}(p) H$-module, then its restriction to a subgroup of $H$ is also projective [5, Theorem VII. 7.11(a)].

To prove the other direction, we use the main theorem from [1] which shows that, for a soluble group $H$ and an algebraically closed field $E$, an $E H$-module is primitive if and only if it is quasi-primitive.

Assume that $V$ is not projective. Since $V$ is not projective, $V_{E}$ is not projective. On the other hand, since $V$ is semisimple and the semisimplicity of $V$ is retained when changing fields (by [5, Theorem VII, 1.8]), $V_{E}$ is semisimple. Since $V_{E}$ is not projective, there exists a simple direct summand $U$ of $V_{E}$ which is not projective. Furthermore, since $H$ is soluble and $U$ is a simple $E H$-module, applying [1, main theorem], we deduce that there is a primitive $E A$-module $W$ where $A \leq H$ and $U \cong W^{H}$ ( $A$ is called a stabilizer limit for $\left.U\right)$. We have that $W$ is simple as an $A$-module by definition, because it is primitive.

First we show that if $X$ is a subgroup of $A$ having order $p$, then $W_{X}$ is projective. Let $X$ be a subgroup of $A$ of order $p$. Since $U \cong W^{H}$, we have $U_{X} \cong\left(W^{H}\right)_{X}$. On the other hand, let $\left\{1, g_{2}, \ldots, g_{m}\right\}$ be a full set of $(A, X)$-double coset representatives of $H$. Applying Mackey's theorem [2, Theorem B. 6.20], we see that $\left(W^{H}\right)_{X}$ is isomorphic to the tensor product

$$
\left((W \otimes 1)_{A \cap X}\right)^{X} \oplus\left[\bigoplus_{i=2}^{m}\left(\left(W \otimes g_{i}\right)_{A^{g_{i}} X}\right)^{X}\right] .
$$

But observe that

$$
\left((W \otimes 1)_{A \cap X}\right)^{X}=\left(W_{X}\right)^{X}=W_{X} .
$$

As $U_{X} \cong\left(W^{H}\right)_{X}$ is projective by assumption, $W_{X}$ is also projective by [2, Proposition B. 2.4]. Since $W_{X}$ is projective, it has dimension divisible by $p$. By [7, note after Theorem 12], the dimension of $W$ is coprime to $p$. Therefore $A$ does not contain any subgroup of order $p$ and so $W$ is projective as an $E A$-module. By [2, Proposition B, 6.12], $W^{H}$ is projective. Hence, $U$ is projective since $U \cong$ $W^{H}$. Therefore $V_{E}$ is projective and $V$ is also projective, which gives the final contradiction.

\section{Proof of main theorem}

Suppose that $G=N H$ where $N$ is a $p$-group. Suppose first that $N$ is not projective as a $\mathrm{GF}(p) H$-module. By Lemma 9, there exists a subgroup $H_{0}$ of $H$ of order $p$ such 
that $N$ is not projective as a $\mathrm{GF}(p) H_{0}$-module. By [2, Theorem B. 4.12] and since $N_{H_{0}}$ is not projective, we know that $N_{H_{0}}$ is not free. Now by Corollary 7 , the number of subgroups of order $p$ in $\mathrm{NH}_{0}$ that intersect $N$ trivially is different from the number of conjugates of $H_{0}$ in $\mathrm{NH}_{0}$. Therefore by Lemma 8, there exists a partial complement of $N$ in $G$ of order $p$ which is not contained in a complement of $N$ in $G$.

We now suppose that $N$ is projective as a $\mathrm{GF}(p) H$-module. Let $K$ be a partial complement of $N$ in $G$. We find a subgroup $H_{0} \leq H$ such that $K$ and $H_{0}$ are both complements of $N$ in $N K$. By Dedekind's lemma [2, Lemma A. 1.3],

$$
N(N K \cap H) \cong(N K \cap N H)=N K .
$$

Observe that, by the isomorphism theorems,

$$
(N K \cap H) \cong N(N K \cap H) / N \cong N K / N \cong K,
$$

since $K$ and $N$ have trivial intersection. Hence $(N K \cap H)=H_{0}$ for some $H_{0} \leq H$ where $K \cong H_{0}$.

By [5, Theorem VII. 7.11(a)], $N$ as a $\mathrm{GF}(p) H_{0}$-module is projective. Furthermore, by [3, Section 2.2], all cohomologies (in particular, the first) vanish. On the other hand, by [2, Theorem A. 15.10], the number of conjugacy classes of complements of $\mathrm{NH}_{0}$ is the order of the first cohomology group and so all complements are conjugate. Now $H_{0}$ and $K$ are both partial complements of $N$ in $N H_{0}$ so $K=H_{0}^{n}$ for some $n$ in $N$. As a consequence, $K=H_{0}^{n} \leq H^{n}$. That is, $K$ is in a complement of $N$ in $G$. Thus we have proved the theorem when $N$ is a $p$-group.

Now let $N=N_{p^{\prime}} N_{p}$ and $G=N H$. Observe that $H$ is a complement of $N$ in $G$ if and only if $N_{p^{\prime}} H$ is a complement of $N_{p}$ in $G$. Firstly, all subgroups $N_{p^{\prime}} H^{n}$ are complements of $N_{p}$ in $G$. Secondly, assume that $C$ is any complement of $N_{p}$ in $G$. Let $q$ be a prime which divides the order of $N_{p^{\prime}}$. Since the index of $C$ is prime to $q$, there is a Sylow $q$-subgroup of $G$ in $C$, and as a consequence $N_{q} \leq C$. Since this holds for any prime $q$ that divides the order of $N_{p^{\prime}}, N_{p^{\prime}} \leq C$. Now we have to show that all the complements of $N_{p}$ are conjugate. First observe that

$$
C=C \cap N_{p^{\prime}} N_{p} H=N_{p^{\prime}}\left(C \cap N_{p} H\right) \quad \text { and } \quad N_{p^{\prime}} \cap\left(C \cap N_{p} H\right)=1 .
$$

Therefore $C \cap N_{p} H$ is a complement of $N_{p^{\prime}}$ in $C$ and so $C \cap N_{p} H$ is a complement of $N_{p} N_{p^{\prime}}$ in $G$. Therefore $C \cap N_{p} H$ is a conjugate of $H$, and as a consequence all the complements of $N_{p}$ in $G$ are conjugates of $N_{p^{\prime}} H$.

By [2, Theorem B, 4.11] if $N_{p}$ is projective as a $\mathrm{GF}(p) H$-module then $N_{p}$ is projective as a $\mathrm{GF}(p) N_{p^{\prime}} H$-module. By [5, Theorem VII. 7.11(a)] if $N_{p}$ is projective as a $\mathrm{GF}(p) N_{p^{\prime}} H$-module then $N_{p}$ is projective as a $\mathrm{GF}(p) H$-module. Therefore $N_{p}$ is projective as a $\operatorname{GF}(p) H$-module if and only if $N_{p}$ is projective as a $\operatorname{GF}(p) N_{p^{\prime}} H$ module. Hence $G$ and $N_{p}$ satisfy the hypothesis of the theorem.

Now suppose that, for each prime $p$ that divides the order of $N$, the Sylow $p$ subgroup $N_{p}$ is projective as a $\mathrm{GF}(p) N_{p}^{\prime} H$-module (that is, $N_{p}$ is projective as a $\mathrm{GF}(p) H$-module). By [6, Corollary 5] and the $p$-group case, every partial complement of $N$ in $G$ is contained in a complement of $N$ in $G$. 
Now suppose that there exists a prime $p$ that divides the order of $N$ such that the Sylow $p$-subgroup $N_{p}$ is not projective as a $\operatorname{GF}(p) N_{p}^{\prime} H$-module and so $N_{p}$ is not projective as a $\operatorname{GF}(p) H$-module. By [6, Corollary 5] and the $p$-group case, there exists a partial complement of $N$ in $G$ that is not contained in a complement of $N$ in $G$. We have thus proved Theorem 1 .

\section{References}

[1] T. R. Berger, 'Primitive solvable groups', J. Algebra 33 (1975), 9-21.

[2] K. Doerk and T. Hawkes, Finite Soluble Groups (Walter de Gruyter, Berlin, 1992).

[3] K. W. Gruenberg, Cohomological Topics in Group Theory, Vol. 143 (Springer, Berlin, 1970).

[4] P. Hall, 'Theorems like Sylow's', Proc. London Math. Soc. (3) 6 (1956), 286-304.

[5] B. Huppert and N. Blackburn, Finite Groups II (Springer, Berlin, 1982).

[6] J. S. Rose, 'Conjugacy of complements in relative holomorphs of finite abelian groups', J. Lond. Math. Soc. (2) 16 (1977), 437-448.

[7] D. Suprunenko, Soluble and Nilpotent Linear Groups (American Mathematical Society, Providence, RI, 1963).

INGRID CHEN, Mathematical Sciences Institute, The Australian National University, Canberra, ACT 0200, Australia

e-mail: ingrid.chen@anu.edu.au 